Results 12 children (13.3\%) were hospitalized during the first days, in the first 3 days - 32 children (35.5\%). In 90 children (100\%) was identificate the antigen of rotavirus. Most frequency of RI in the children was identificated in age 1-3 years. The syndrome of gastroenteritis developed in 1-3 day per acute period of disease Frequency of stool was $2-15$ times per days. In the 20 children $(22.2 \%)$ RI was associated with pathogenic flora: St. aureus (7.7\%), Proteus $(10 \%)$, Pseudomonas (4.4\%). In the our contingent there were a decline of levels of copper of blood serum, zinc and iodine, at the insignificant increase of level of iron. Research of mineraluria in children gave the reference level of all investigated oligoelementss and phosphorus. In the patients with RI was identificated the disbalance of minerals of blood serum with association of their unchanged elimination.

Conclusions This dates presents the necessity of mineral ocorrection in the patients with RI.

\section{GENETIC AND PHYLOGENETIC CHARACTERIZATION OF STAPHYLOCOCCUS AUREUS STRAINS ISOLATED FROM ATOPIC DERMATITIS PEDIATRIC PATIENTS AND THEIR COHABITANTS}

doi:10.1136/archdischild-2012-302724.0918

${ }^{1} \mathrm{~S}$ Pecetta, ${ }^{2} \mathrm{C}$ Pascolini, ${ }^{2} \mathrm{G}$ Prignano, ${ }^{2} \mathrm{~F}$ Ensoli, ${ }^{2} \mathrm{~B}$ Capitanio, ${ }^{1} \mathrm{C}$ Passariello. 'Department of Public Health and Infectious Diseases, University 'La Sapienza'; ' Clinical Pathology and Microbiology Laboratory and Pediatric Dermatology Division, IFO-IRCCS San Gallicano, Rome, Italy

Atopic dermatitis $(\mathrm{AD})$ is characterized by dysfunctional skin susceptible to Staphylococcus aureus colonization, which can exacerbate the symptoms. Recent studies indicate that the $S$. aureus's great versatility is direct consequence of its genome's plasticity and adaptability.

The present study evaluated the prevalence of $S$. aureus in 175 pediatric $\mathrm{AD}$ patients and their 195 cohabitants in relation with the severity of the disease. Moreover, isolated strains were characterized for pathogenic and virulence factors (PCR analysis), for genome structure (PFGE analysis) and for phylogenetic relations (MLST analysis) to investigate the possible correlation between genetic characteristics and the different stages of disease and the effects of atopic environment on the genome structure of these strains.

Our data showed that both patients and their cohabitants had high prevalence of $S$. aureus, that was proportional to the severity of the disease. PFGE analysis showed the existence of clonal identity among isolates from different sites of the same patient and between isolates from patients and their cohabitants. MLST data showed that there was a significant phylogenetic distance among strains with identical PFGE profile.

Our results demonstrate that the family is a source of infection/ reinfection for patients and a source of risk for cohabitants. Moreover, our data suggests that although bacterial strains from atopic skin show conserved genomic structures (identical PFGE profiles), they came from very different genetic backgrounds (different MLST profiles). We assume that the peculiar atopic tissue environment may induce the evolution of these strains, with changes in genomic structure and regulation of virulence factors.

\section{HUMAN BOCAVIRUS IN HIGH-RISK CHILDREN}

doi:10.1136/archdischild-2012-302724.0919

'S Al-Hajjar, ${ }^{2}$ S Al-Thawadi, ${ }^{3} \mathrm{~A}$ Al-Seraihy, 'I Bin-Hussain. 'Pediatrics; ${ }^{2}$ Pathology and Laboratory Medicine; ${ }^{3}$ Pediatric Hematology-Oncology, King Faisal Specialist Hospital \& Research Centre, Riyadh, Saudi Arabia

From 1 October 2009 to 1 December 2010, we conducted a prospective hospital-based study at KFSHRC to evaluate the role of Bocavirus $(\mathrm{HBoV})$ infections in hospitalized children with chronic medical or immunocompromising conditions. Clinical and epidemiological data were recorded and respiratory samples including nasopharyngeal aspirate or nasopharyngeal swabs were obtained from all children less than 14 years old with acute respiratory tract infections. HBoV was screened in all respiratory samples by real time PCR, in addition to 13 common respiratory viruses. During the study, HBoV was detected in respiratory samples from $25(2 \%)$ of 1016 symptomatic patient. HBoV co-existence with other respiratory pathogens occurred in $72 \%(18 / 25)$ of respiratory samples from symptomatic patients. HBoV infections were detected in every month except June and July with peaks in the month of September, October, November, and December. The main diagnosis in 13 patients $(52 \%)$ with $\mathrm{HBoV}$ was radiologically confirmed pneumonia. For the other 12 patients with $\mathrm{HBoV}$ infections the main diagnosis were gastroenteritis (4 cases), chest exacerbation (3 cases), upper respiratory tract infections (2 cases), persistent fever (1 case), seizure (1 case), otitis media ( 1 case). The main clinical signs and symptoms of $\mathrm{HBoV}$ positive patients included fever, cough tachypnea, dyspnea, crackles, wheezing, abdominal pain, vomiting and diarrhea. The present study suggest that $\mathrm{HBoV}$ may be a fairly common cause of pneumonia in high-risk children hospitalized with acute respiratory infections and associated with morbidity. However, further study is needed to clarify if $\mathrm{HBoV}$ plays a pathogenic role in community acquired pneumonia in high-risk children.

\section{SIMILAR CLONE OF SALMONELLA ENTERICA SEROVARES ENTERITIDIS ISOLATED FROM STOOL SAMPLES OF CHILDREN AND FOOD SOURCES}

doi:10.1136/archdischild-2012-302724.0920

'MM Soltan Dallal, ' $\mathrm{F}$ Fardsanei, ${ }^{2} \mathrm{M}$ Saifi, ${ }^{2} \mathrm{E}$ Jabbarzadeh. 'Division of Microbiology, School of Public Health, Tehran University of Medical Science; 'Department of Microbiology, Pasteur Institute of Iran, Tehran, Iran

Background Salmonella enterica subsp. Enterica serovar enteritidis is one of the major endemic causes of gastroenteritis worldwide. The objective of this study was to molecular analysis of Salmonella enteritidis (SE) isolates from children stools and food sources.

Methods During 6 months (2010), 1950 stool samples of children younger than 12 years in Tehran were collected. At the same time sampling from different food sources including chicken, beef, lamb and duck also, were done. The culture and susceptibility testing performed according to the standard methods. We also used rep-PCR with $(\mathrm{GTG})_{5}$ primers to study genetic relatedness of SE isolates from two sample sources.

Results A total of 30 SE isolates (15 of clinical and 15 of food samples) were identified. There were 14 different antibiotypes (AB) among stool and food sample isolates. AB1 (30\%) and AB2 (24\%) was the most prevalent antibiotic resistance patterns. The (GTG) PCR banding pattern analysis reviled 3 different common types (CT1, CT2, CT3) and only one single type. CT1 and CT2 were shared between food and stool samples and CT2 was predominant clone. CT3 was limited only to the clinical samples.

Conclusion Antibiotic resistance of SE isolates from clinical and food sources did not differ significantly except for nitrofurantion and nalidixic acid. Food sources isolates were more susceptible than clinical ones. Using rep-PCR with (GTG) ${ }_{5}$ primers, showed that some clones of SE are responsible for salmonellosis between human and food sources in Tehran and is of major public health concern.

921 VIRULENCE FACTORS OF UROPATHOGENIC AND COMMENSAL ESCHERICHIA COLI PATHOTYPES IN PATIENTS WITH URINARY TRACT INFECTION

doi:10.1136/archdischild-2012-302724.0921 